Recepción: 07/ 08 / 2018

Aceptación: 25/ 09 / 2018

Publicación: 01 / 11 / 2018

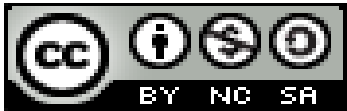

Ciencias económicas y empresariales

Artículo de investigación

\title{
Manual de procedimientos: herramienta de mejora en la productividad de la Empresa Lanafit S.A.
}
Procedural handbook: improvement tool in the productivity of the Lanafit Company S.A.
Procedural handbook: improvement tool in the productivity of the Lanafit Company S.A.

Delia I. Andrango-Guayasamín ${ }^{\mathrm{I}}$ delia.andrango@gmail.com Raúl H. Andrango-Guayasamín II raúl.andrango@utc.edu.ec Edison P. Salazar-Cueva ${ }^{\text {III }}$ edison.salazar@utc.edu.ec Jaime H. Acurio-Masabanda IV Jaime.acurio@utc.edu.ec

\section{Correspondencia: delia.andrango@gmail.com}

\footnotetext{
I Docente Universitario, Instituto Tecnológico Superior Gran Colombia, Quito, Ecuador.

II Docente de la Universidad Técnica de Cotopaxi, Latacunga, Ecuador.

III Magíster en Seguridad y Prevención de Riesgos del Trabajo, Ingeniero Industrial, Tecnólogo en Mecánica AeronáuticaEstructuras, Docente de la Universidad Técnica de Cotopaxi, Latacunga, Ecuador.

IV Magíster en Tecnologías para la Gestión y Práctica Docente, Licenciado en Ciencias de la Educación Mención Psicopedagogía Infantil, Ingeniero en Sistemas, Tecnólogo en Programación De Sistemas, Docente de la Universidad Técnica de Cotopaxi, Latacunga, Ecuador.
} 


\title{
Resumen
}

Una de las formas de garantizar la productividad y efectividad en los procesos productivos de una empresa, consiste fundamentalmente en la creación de herramientas necesarias para realizar el seguimiento las labores y responsabilidades inherentes a dichos procesos. La investigación consistió en analizar los procesos actuales de confección y la elaboración de un manual de procedimientos que garantice los procesos productivos en pro de alcanzar y solidificar la eficiencia, credibilidad, eficacia y así mismo consolidar todos los servicios. La investigación se desarrolla para mejorar la productividad a través de una estandarización de operaciones. El estudio se plantea como una investigación no experimental en la que adopta una modalidad de campo apoyándose en una investigación bibliográfica documental que permite evaluar el sistema productivo en las empresas para posteriormente mejorar la situación actual mediante la elaboración de una propuesta, analizando casos prácticos que garantice la reducción de los procesos y los costos operacionales. Como resultado se identificó los problemas operacionales en cada etapa del proceso, así como la baja productividad debido a la carencia de instructivos para la elaboración de prendas. Finalmente se concluye que los procesos de confección presentan particularidades en su ejecución, el uso de instructivos de trabajo que permiten reducir los costos operativos y aumentar la productividad de manera que mejoren las estadísticas de la empresa Lanafit S.A. en Ecuador.

Palabras clave: manual de procedimientos; productividad; empresa.

\begin{abstract}
One of the ways to guarantee the productivity and effectiveness in the productive processes of a company, consists fundamentally in the creation of necessary tools to carry out the follow-up of the tasks and responsibilities inherent to said processes. The research consisted of analyzing the current manufacturing processes and the preparation of a procedures manual that guarantees the productive processes in order to achieve and solidify the efficiency, credibility, effectiveness and likewise consolidate all the services. The research is developed to improve productivity through standardization of operations. The study is proposed as a non-experimental research in which it adopts a field modality supported by a bibliographic documentary research that allows to evaluate the productive system in companies to subsequently improve the current situation by preparing a proposal, analyzing practical cases that guarantee the reduction of processes and operational
\end{abstract}


costs. As a result, the operational problems were identified in each stage of the process, as well as the low productivity due to the lack of instructions for the elaboration of garments. Finally, it is concluded that the manufacturing processes present particularities in their execution, the use of work instructions that reduce operating costs and increase productivity in a way that improves the statistics of the company Lanafit S.A. in Ecuador.

Key words: procedures manual; productivity; company.

\section{Resumo}

Uma forma de garantir a produtividade ea eficiência nos processos de produção de uma empresa, consiste basicamente na criação de ferramentas necessárias para monitorar as tarefas e responsabilidades inerentes a tais processos. A pesquisa envolveu a análise de processos de fabricação atuais e o desenvolvimento de um manual de procedimentos para garantir processos de produção para alcançar e solidificando eficiência, credibilidade, eficiência e também consolidar todos os serviços. A pesquisa é desenvolvida para melhorar a produtividade através da padronização de operações. O estudo é apresentado como uma pesquisa não-experimental que adota um campo modo de contar com um documentário literatura que avalia o sistema produtivo em empresas para melhorar ainda mais a situação atual através do desenvolvimento de uma proposta, analisando estudos de caso para garantir a redução de processos e custos operacionais. Como resultado de problemas operacionais em cada fase do processo que foi identificado e baixa produtividade devido à falta de instruções para fazer vestuário. Finalmente concluiu que os processos de fabricação têm particularidades em execução, o uso de instruções de trabalho que reduzem os custos operacionais e aumentar a produtividade de modo a melhorar as estatísticas da empresa Lanafit S.A no Equador.

Palavras chave: manual de procedimentos; produtividade companhia.

\section{Introducción}

Un manual de procedimientos es un método o manera de proceder en algunos procesos, ciclo de operaciones que afectan a varios empleados que trabajan en sectores distintos y que se establece para asegurar el tratamiento uniforme de todas las operaciones respectivas para producir un determinado bien o servicio. Dávila Palacios (2017). Estos manuales de procesos incluye una 
serie de políticas, normas y condiciones que permiten el correcto funcionamiento de la empresa, de esta manera están preparados ante la complejidad y los problemas cada día mayores que presenta la moderna industria de la confección, tanto en las industrias pequeñas, medianas o grandes han ido creando en las personas vinculadas a este campo como dueños y directivos, la necesidad de adquirir conocimientos y experiencias en los aspectos relacionados con las distintas técnicas, métodos y herramientas textiles, para desarrollarse en un mercado cada vez más competitivo y de condiciones variables.

Cabe destacar que la empresa Lanafit SA se encuentra dentro del grupo de organizaciones que ofertan prendas formales en la ciudad de Quito y Guayaquil, es así como desde hace varios años ha bajado su nivel competitivo dentro del mercado, desestimando las bondades que giran en torno a un manual de procesos de manera eficiente, para la confección en sus prendas y el incontenible avance de la industria están siendo los factores para que esta organización vaya perdiendo la posición que había alcanzado en algún momento, las circunstancias mencionadas hace conveniente la estandarización de procedimientos en el proceso de confección, con la finalidad de tener un orden en cada actividad que involucre la elaboración de prendas. Es así como el propósito de esta investigación consistió en analizar los procesos actuales de confección y la elaboración de un manual de procedimientos que garantice el proceso productivo en pro de alcanzar y solidificar la eficiencia, credibilidad, eficacia y así mismo consolidar todos los servicios. La investigación se desarrolla para mejorar la productividad a través de una estandarización de operaciones, de esta manera se cumpliría con la misión planteada por la organización que es entregar prendas de calidad a sus clientes, puntualidad y seriedad en sus contratos, además de volver a obtener alta posición dentro de la industria de la confección.

\section{Desarrollo del Tema.}

Este tema pretende abarcar los procesos inherentes a un manual de producción, por tanto, el manual es conceptualizado como una recopilación informa recopilación en forma de texto, que recoge minuciosa y detalladamente las instrucciones que se deben seguir para realizar una determinada actividad, de una manera sencilla y adaptable a la comprensión de los interesados. Todo manual de procedimientos aspira documentar la experiencia, el conocimiento y aquellas técnicas que de una u otra forma se generan en un organismo; considerándose de esta manera una 
herramienta que suma las experiencias y técnicas requeridas para un proceso determinado (para este estudio seria para un proceso de producción. Por tanto, un manual de procedimientos contribuye a enfocar los esfuerzos y la atención de los integrantes de una organización hacia la mejora de los sistemas de trabajo y su nivel de competitividad. De esta manera es necesario tomar en cuenta la eficiencia, definida como la mejor utilización de los recursos, técnicos, materiales, humanos y financieros con el fin de mejorar las condiciones de salud de la población atendida y además la calidad que está relacionada con la atención efectiva, oportuna, personalizada, humanizada, continua, de acuerdo con estándares aceptados sobre procedimientos Científico así como los Técnicos y Administrativos mediante la utilización de la tecnología apropiada, de acuerdo con los requerimientos de los servicios que pretenda la empresa considerar en busca de mojaras en los procesos.

\section{Metodología}

La metodología empleada para el estudio, fue de carácter descriptiva y con diseño de campo, las mismas se apoyaron en fuentes documentales que fueron bases para el desarrollo y comprensión de la temática.

Cabe destacar que la investigación se desarrolló sin manipular las variables, por lo cual se empleó se empleó el método de la observación el cual tiene la capacidad de describir y explicar el comportamiento requerido para la obtención de datos adecuados y fiables correspondientes a las conductas y eventos con situaciones perfectamente identificables por el investigador a través del apoyo de fuentes documentales, a tal efecto se obtuvieron una serie de datos y registros que permitieron a su vez establecer procedimientos de carácter práctico en la mejora de la productividad d ella empresa.

En este sentido, se realizó un diagnóstico del sistema productivo de la referida empresa, el cual consistió en el reconocimiento de elementos tales como la producción del proceso, tamaño, ciclo de demora, tiempos, especialización rendimientos entre otros, al identificar estos factores como párate del proceso productivo, se pudo constatar los sistemas que utiliza la empresa LANAFIT, en consecuencia, la tabla 1 determina sistemáticamente dichos elementos y dichos sistemas. 
Tabla 1. Diagnóstico del Sistema Productivo Actual

\begin{tabular}{|l|l|}
\hline \multicolumn{2}{|c|}{ SISTEMA PRODUCTIVO LANAFIT S.A. } \\
\hline ELEMENTOS & SISTEMA QUE UTILIZA \\
\hline Producción en proceso & Entre 300 unidades semanales \\
\hline Tamaño del lote & 60 unidades \\
\hline Flujo del proceso & Inexistente \\
\hline Ciclo que demora la producción & 5 días \\
\hline Tiempos de cargue de línea & Similar al ciclo de producción \\
\hline Especialización del operario & Polivalentes \\
\hline Rendimiento individual & Bueno \\
\hline Rendimiento grupal & Bueno \\
\hline Calidad de lo confeccionado & Bueno \\
\hline Flexibilidad del equipo & Estático \\
\hline Ausentismo & Bajo \\
\hline Nivel de cooperación del operario & Medio \\
\hline Cumplimiento de los despachos & Bueno \\
\hline Elaboración de especificaciones de \\
calidad
\end{tabular}

Fuente: Andrango, Andrango, Salazar y Acurio (2018) 
Evaluando estos procesos que permiten periódicamente y de manera sistemática determinar en forma objetiva, la relevancia, eficacia, eficiencia estos parámetros se utilizaron para el análisis del proceso que se maneja actualmente. Sin embargo, es importante destacar que aun cuando un gran porcentaje luce como bueno, la falta de instructivo de trabajo (manual de procedimientos) ha ocasionado que el personal de operaciones no tenga claro sus actividades, lo cual conlleva a la baja productividad en la línea de producción.

\section{Resultados}

Considerando la aplicación de los métodos y técnicas desarrollados por la investigación y respecto a la evaluación de los procesos productivos, con énfasis en la confección de prendas (traje formal-dama) y su relación a la baja productividad de las empresas. Se analizó el proceso de confección actual en la empresa, recopilando información de las operaciones que influyen en la elaboración de trajes formales para dama, mediante el uso de instrumentos como la observación, revisión documental y bibliográfica obteniendo como resultado las deficiencias en cada etapa de operación que deben mejorarse.

También se logró identificar las falencias operacionales que inciden en la baja productividad de la organización, debido a que la ficha técnica, el flujo de procesos y la distribución del módulo; no muestran un orden consecutivo y controlado para la línea de producción. A través de una organización en las operaciones se mejorará la eficiencia productiva. De esta manera la evaluación del proceso de confección se fundamenta en mejorar los procesos operacionales por medio de la organización de los procedimientos para llegar a cumplir un nivel de producción alto y maximizar la rentabilidad. Es así como con estos antecedentes se establece un manual de procedimientos para que sea estandarizado entre los operarios y permita la confección de prendas de manera ordenada, reduciendo los procesos e incrementando la productividad.

La tabla No 2 establece un plan de acción con respecto a los procesos inherente de producción que identifica los propósitos a seguir, la estrategia, los responsables, los recursos y la meta correspondiente. 
Tabla 2. Plan de acción "Producción"

\begin{tabular}{|c|c|c|c|c|}
\hline \multicolumn{5}{|c|}{ PLAN DE ACCIÓN "PRODUCCIÓN" } \\
\hline \multicolumn{5}{|c|}{$\begin{array}{l}\text { EMPRESA LANAFIT S.A. } \\
\end{array}$} \\
\hline OBJETIVO & ESTRATÉGIA & RESPONSABLE & RECURSOS & META \\
\hline $\begin{array}{l}\text { Implementar } \\
\text { los } \\
\text { procedimientos } \\
\text { del proceso de } \\
\text { confección }\end{array}$ & $\begin{array}{l}\text { Estandarizar y } \\
\text { comprometer a todos los } \\
\text { que conforman la } \\
\text { empresa }\end{array}$ & $\begin{array}{l}\text { Jefe de sección } \\
\text { Jefe de } \\
\text { producción }\end{array}$ & $\begin{array}{l}\text { Técnicos y factor } \\
\text { humano de la } \\
\text { empresa }\end{array}$ & $\begin{array}{l}\text { Entregar al } \\
\text { mercado } \\
\text { prendas de } \\
\text { calidad a un } \\
\text { tiempo } \\
\text { oportuno }\end{array}$ \\
\hline $\begin{array}{l}\text { Capacitar al } \\
\text { personal }\end{array}$ & $\begin{array}{l}\text { Instruir a todo el } \\
\text { personal sobre procesos, } \\
\text { calidad y mantenimiento }\end{array}$ & $\begin{array}{l}\text { Jefe de } \\
\text { producción }\end{array}$ & $\begin{array}{l}\text { Técnicos y factor } \\
\text { humano de la } \\
\text { empresa }\end{array}$ & $\begin{array}{l}\text { Procesos } \\
\text { operativos }\end{array}$ \\
\hline $\begin{array}{l}\text { Realizar estudio } \\
\text { de métodos y } \\
\text { tiempos }\end{array}$ & $\begin{array}{l}\text { Delegar y capacitar al } \\
\text { jefe de producción } \\
\text { Estandarizar los tiempos } \\
\text { Registrar los tiempos } \\
\text { tomados en los formatos } \\
\text { correspondientes }\end{array}$ & $\begin{array}{l}\text { Jefe de } \\
\text { producción }\end{array}$ & $\begin{array}{l}\text { Cronómetro } \\
\text { Tabla de tiempos } \\
\text { Hoja de tiempos }\end{array}$ & $\begin{array}{l}\text { Obtener los } \\
\text { tiempos de } \\
\text { confección } \\
\text { de las } \\
\text { prendas para } \\
\text { mejorar la } \\
\text { planificación } \\
\text { y } \\
\text { productividad }\end{array}$ \\
\hline $\begin{array}{l}\text { Organizar } \\
\text { reuniones de } \\
\text { trabajo }\end{array}$ & $\begin{array}{l}\text { Realizar reuniones entre } \\
\text { la gerencia y la planta de } \\
\text { producción }\end{array}$ & $\begin{array}{l}\text { Jefe de sección } \\
\text { Jefe de } \\
\text { producción } \\
\text { Gerencia }\end{array}$ & $\begin{array}{l}\text { Oficina gerencia } \\
\text { Sala de reuniones }\end{array}$ & $\begin{array}{l}\text { Llevar un } \\
\text { seguimiento } \\
\text { de los } \\
\text { procesos } \\
\text { operativos y } \\
\text { de control }\end{array}$ \\
\hline $\begin{array}{l}\text { Registrar el } \\
\text { incremento de } \\
\text { la calidad }\end{array}$ & $\begin{array}{l}\text { Desarrollar el proceso de } \\
\text { inspección de calidad } \\
\text { haciéndoles partícipes a } \\
\text { los operarios en el } \\
\text { estudio técnico }\end{array}$ & $\begin{array}{l}\text { Jefe de sección } \\
\text { Jefe de } \\
\text { producción }\end{array}$ & $\begin{array}{l}\text { Indicadores de } \\
\text { calidad }\end{array}$ & $\begin{array}{l}\text { Disminuir re } \\
\text { procesos }\end{array}$ \\
\hline $\begin{array}{l}\text { Desarrollar el } \\
\text { potencial del } \\
\text { personal }\end{array}$ & $\begin{array}{l}\text { Convivencia humana, } \\
\text { ambiente de trabajo }\end{array}$ & $\begin{array}{l}\text { Jefe de sección } \\
\text { Jefe de } \\
\text { producción } \\
\text { Gerencia }\end{array}$ & Conferencistas & $\begin{array}{l}\text { Fortalecer el } \\
\text { trabajo en } \\
\text { equipo } \\
\text { Motivar al } \\
\text { operario para } \\
\text { un mayor } \\
\text { aporte en la } \\
\text { producción }\end{array}$ \\
\hline
\end{tabular}

Fuente: Andrango, Andrango, Salazar y Acurio (2018) 
De igual forma se destaca el punto muerto y/o umbral de rentabilidad (en inglés break-even point - BEP) el cual se define como el número mínimo de unidades que una empresa necesita vender para que el beneficio en ese momento sea cero. Se muestran las unidades con sus costos fijos, variables, medios, ingresos y beneficios. Identificándose así mismo en la última columna de la figura 1 un saldo negativo respecto a las unidades y costos señalados.

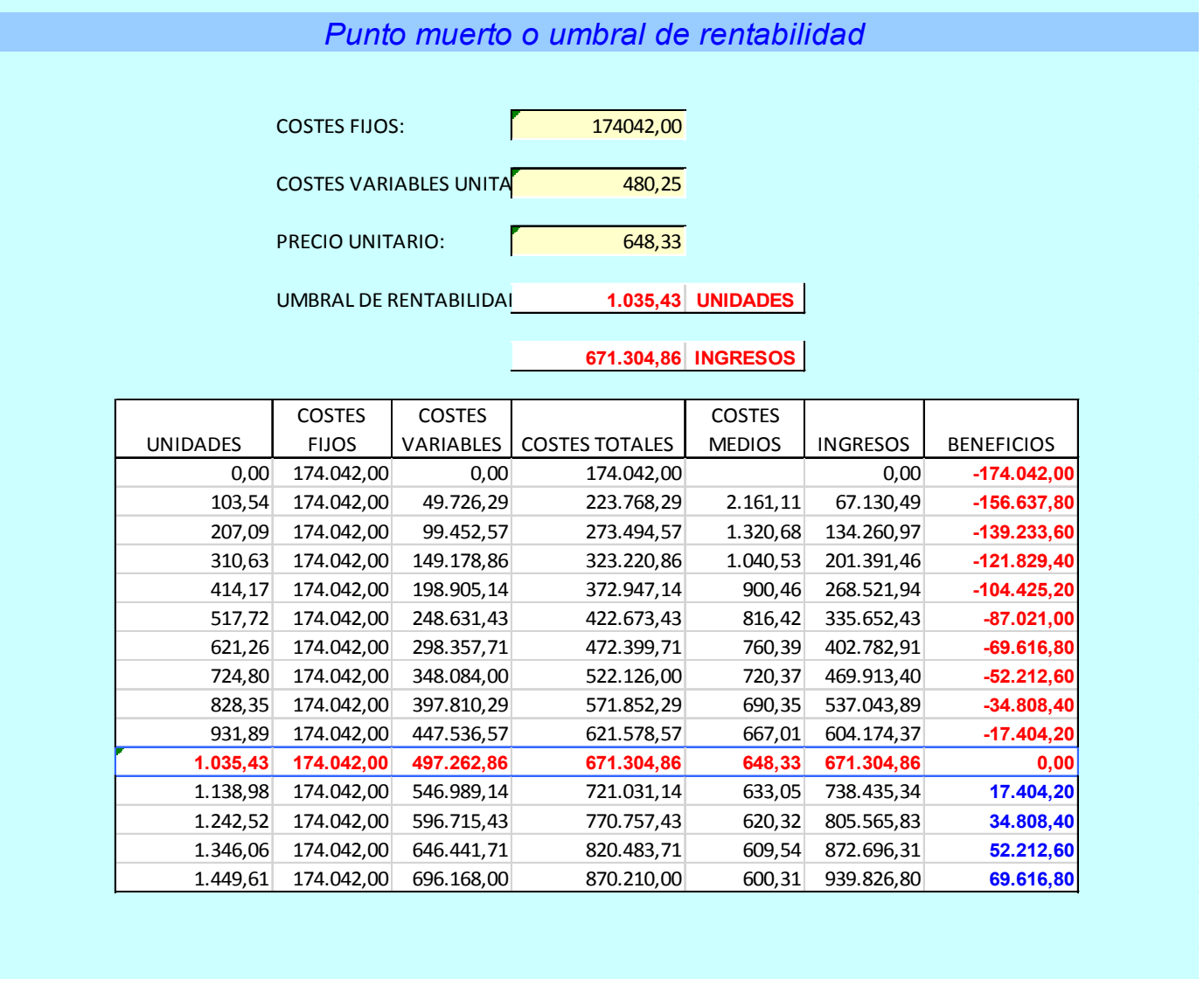

Figura 1: Punto muerto o umbral de rentabilidad

Fuente: Andrango, Andrango, Salazar y Acurio (2018)

El grafico 1 señala el punto de equilibrio de los costos con antelación siendo de igual manera una herramienta clave en la estrategia de una empresa, dado que es fundamental para ponderar el grado de solvencia de una empresa y su potencial de rentabilidad. 


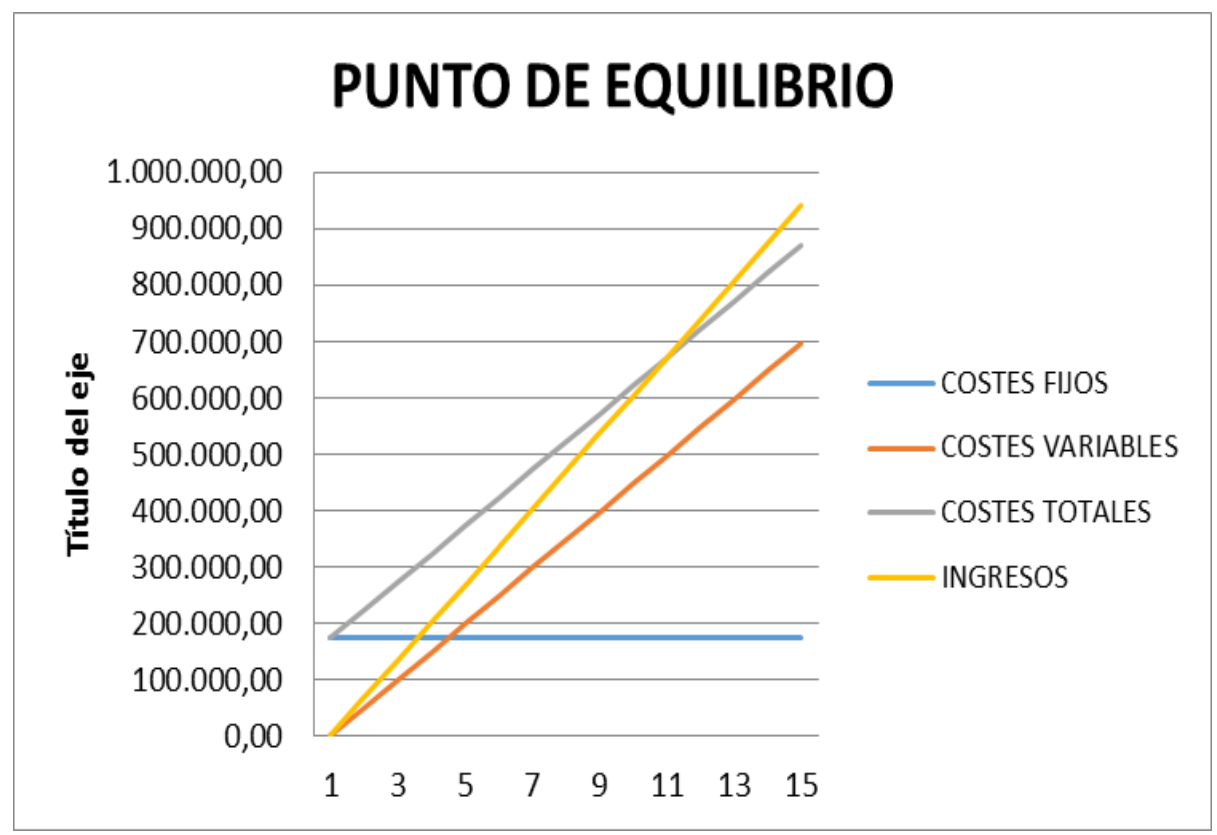

Gráfico 1: Demostración gráfica del punto de equilibrio

Fuente: Andrango, Andrango, Salazar y Acurio (2018)

\section{Conclusiones}

El trabajo titulado MANUAL DE PROCEDIMIENTOS: HERRAMIENTA DE MEJORA EN LA PRODUCTIVIDAD DE LA EMPRESA LANAFIT S.A. busco analizar el proceso productivo que determina los niveles de calidad y eficiencia en la empresa objeto de estudio, a tal efecto es relevante destacar que todo manual de procedimiento, constituye la base del sistema de calidad así como las mejoras continuas en la búsqueda de la competitividad, no se trata de establecer normas y procedimientos que no se ajusten en forma específica a los sistemas productivos, se trata de establecer los parámetros que hacen que la organización sea mucho más competitiva y adecuada al contexto actual. Entre los aspectos conclusivos del trabajo cabe señalar los siguientes:

Mediante una observación de campo en la empresa Lanaria S.A., se realizó la descripción del proceso actual de confección identificando en cada operación la forma de coser las prendas, para determinar nuevos parámetros de operación que mejoren su rendimiento e incrementen la productividad. 
Durante la observación de la planta con la ayuda de registros de operación e indicadores de producción, se identificó las falencias operacionales presentadas en cada etapa del proceso determinando que existe desorden en las operaciones, lo que conllevan a generar pérdidas.

La implementación de un manual de procesos, permitirá un mayor control del proceso productivo en la línea de prendas de mujer, así como el aumento de la rentabilidad que se verá reflejado en el cumplimiento de metas y ganancias económicas para la empresa.

\section{Referencias Bibliográficas}

Amado N (2005) Prospectiva del sector de Confecciones. Colombia: Editorial Gaia

García, G (2013) Gestión de la Productividad. México: Editorial Mac Graw Hill.

González H (2015= Factores que afectan la productividad. Universidad de Chile. Dpto. de Producción Animal. Documento en Línea file://C:/Users/DELL/Downloads/factores $\% 20$ que $\% 20$ afectan $\% 201 \mathrm{a} \% 20$ productividad $\% 20 \mathrm{de} \% 2$ 01os\%20sistemas\%20pastoriles\%20de\%20produccion $\% 20 \mathrm{de} \% 201$ eche.pdf

Guía de Elaboración de Procedimientos (2018) Documento técnico para la iniciativa de formación en gerencia social y protección social. Zona Sur. Occidente FONADE. Universidad ICESI. Chile.

Manual de Procedimientos (2016) Comisión Estatal para el Acceso a la Información Pública. CEAIP. Estado De Zacatecas. México

Ortiz I (2008) Documento técnico para "la iniciativa de formación en gerencia social para la protección social” zona sur-occidente FONADE- universidad icesi monitoreo y evaluación desarrollo de indicadores 